\title{
La biodegradación de biopolímeros: un aporte a la educación en ciencias para la sustentabilidad en la formación de profesores de química
}

\author{
Biodegradation of Biopolymers: A Contribution to \\ Science Education for Sustainability in the Training \\ of Chemistry Teachers
}

\author{
Ángel Danilo Florián Ardila ${ }^{1}$ \\ Ricardo Andrés Franco Moreno
}

\section{Resumen}

En el presente trabajo de investigación se describe, se estructura y se orienta el diseño de una secuencia didáctica implementada y desarrollada a partir del proceso de elaboración y biodegradación de un biopolímero plástico por la acción de microrganismos fúngicos, como camino hacia el fomento de la educación en ciencias para la sustentabilidad ambiental en la formación de profesores de química, en un curso electivo de química verde.

La implementación de este proyecto de investigación tiene como objetivo fomentar y acercar al profesor de química en formación inicial a plantear soluciones para el mejoramiento de la calidad de vida y del ambiente. La secuencia didáctica se desarrolló bajo el supuesto de tres conceptos principales desarrollados con base en la química verde, los cuales interaccionan a modo de nodos en una red de aprendizaje, que son: contaminación por plásticos, biodegradación y sustentabilidad ambiental en química verde. A partir de la integración de los tres nodos se ha planteado una secuencia didáctica con diversidad de actividades teóricas, socio-críticas y prácticas, haciendo énfasis en la importancia de la educación en ciencias para la sustentabilidad, a través de la implementación de trabajos prácticos de laboratorio que buscan contribuir desde el saber científico a mitigar las problemáticas socioambientales.

1 Estudiante de la Licenciatura en Química de la Universidad Pedagógica Nacional. Correo electrónico: danilo.835@ hotmail.com

2 Docente de la Licenciatura en Química de la Universidad Pedagógica Nacional. Miembro del grupo de investigación IREC. Correo electrónico: rfranco@pedagogica.edu.co 
Palabras clave

Sustentabilidad ambiental; biodegradación; química verde; biopolímeros; trabajos prácticos de laboratorio; secuencia didáctica

\section{Abstract}

This article describes, structures and guides the design of a teaching sequence implemented and developed from the process of preparation and biodegradation of a biopolymer plastics by the action of fungal microorganisms, as a way to promote science education for environmental sustainability in the training of chemistry teachers, in an elective of green chemistry.

The implementation of this research project aims to promote and approach the professor of chemistry in initial training, to propose solutions for the improvement of the quality of life and the environment. The didactic sequence was developed under the assumption of three developed main concepts based on green chemistry, which interact as a node in a network of learning and which are: contamination by plastics, biodegradation and environmental sustainability, in green chemistry. It has been based on the interrelation of these previously mentioned concepts, a sequence didactics with a diversity of theoretical activities, socio-critical and practice, emphasizing the importance of science for sustainability education, through the implementation of practical laboratory work, to contribute from scientific knowledge to mitigate the socio-environmental problems.

\section{Keywords}

Sustainability; biodegradation; education; green; teacher training; plastic biopolymers; practical works of laboratory; environmental pollution; chemical sequence didactics

\section{Introducción}

Los plásticos son los polímeros sintéticos más abundantes en el planeta, hacen parte en la vida cotidiana de las personas; sin embargo, su mala disposición al finalizar su vida útil provoca que miles de estos se depositen en las calles y en los océanos, convirtiéndose en una problemática que afecta el ambiente, los animales y la salud de los humanos.

La biodegradación por microorganismos es el medio por el cual un polímero se puede descomponer en sus moléculas fundamentales, es por ello que el principal objetivo de este proyecto es evaluar el proceso de biodegradación de bioplásticos por la acción de hongos presentes en el suelo, como alternativa para mitigar el impacto socioambiental que es generado por estos residuos.

De igual manera se propone, en el proyecto curricular de Licenciatura en Química de la Universidad Pedagógica Nacional (UPN), abordar en el plan de estudios de la electiva Química Verde, importante en la educación en ciencias para la sustentabilidad, a través de la implementación de una secuencia de enseñanza-aprendizaje a partir de trabajos prácticos de laboratorio y que busca contribuir desde el saber científico a mitigar las problemáticas socioambientales.

En ese sentido, la formación del profesorado de química demanda una perspectiva pertinente, seria y adecuada de teorías, modelos y aplicaciones de la educación para la sustentabilidad, desde espacios académicos como la electiva de Química Verde para el desarrollo de estrategias de orden conceptual y metodológico. Esto se desarrolla en aras de vincular las competencias del futuro docente para que sea capaz de identificar e intervenir, desde la perspectiva educativa, en la problemática ambiental a partir de la relación sociedad-ciencia-naturaleza. Así mismo, se busca aplicar conceptos básicos y principios que le permitan valorar la importancia de los recursos naturales, para realizar intervenciones educativas pertinentes en pro de la conservación del ambiente y la enseñanza de las ciencias.

Con el propósito de fomentar la educación en ciencias para la sustentabilidad en profesores en formación de química, a través del proceso de biodegradación de bioplásticos, se diseñó y se implementó una secuencia didáctica centrada en trabajos prácticos de laboratorio con profesores en formación inicial de la electiva de Química Verde. 
Con esta secuencia se evidenció la importancia del desarrollo de estos proyectos encaminados a mitigar problemáticas ambientales desde la perspectiva educativa en donde juega un papel importante la enseñanza-aprendizaje, así como el desarrollo de valores naturales, culturales, éticos y estéticos, pues inciden en la visión de la ciencia frente a la contaminación ambiental como alternativa para aminorar la problemática ambiental.

\section{Descripción del problema}

Los polímeros plásticos cambiaron la forma de vida. Desde su descubrimiento han intervenido directamente en el desarrollo científico y tecnológico del ser humano; no obstante, su producción continúa a gran escala. Cada día de nuevas y complejas fórmulas moleculares se obtienen plásticos con el fin de ofrecer diversos beneficios y utilidades a los usuarios, los cuales han provocado que estos se acumulen diariamente sin ningún control como residuos desechables que no aportan ningún servicio.

Lo anterior ha generado que estos compuestos no tengan un final sustentable en su disposición y reutilización final, convirtiéndolos en el contaminante más abundante del planeta. Estos materiales están presentes en las profundidades de mares, lagos, ríos o flotando en ellos, así como en los suelos y en los lugares más remotos; en muchos casos son arrastrados por las aguas que circulan cerca, o por las corrientes de aire que lo pueden transportar de un lugar a otro, afectando la fauna marina, terrestre y a los humanos. Esto produce el transporte de compuestos químicos altamente peligrosos que pueden filtrarse en la cadena alimentaria y llegar a diferentes organismos, afectando la salud, la estética y la percepción de limpieza de las comunidades.

Los gobiernos y la industria de los polímeros plásticos se preocupan y esfuerzan por mitigar su impacto ambiental, realizando campañas hacia una buena disposición final de este residuo; sin embargo, a través de la historia estas actividades no muestran un resultado eficaz, siguen presentes en el medio ambiente porque proporcionan bienestar a los usuarios, a las fábricas que se benefician de su uso, consumo, y por su aporte al sistema económico mundial.

La biodegradación de estos materiales depende de la composición química del producto y su degradación es muy lenta. Tardan desde 100 a 1000 años en descomponerse (González y Manhini, 2003); pierden su forma física presentando una falsa degradación, fragmentándose en partes más pequeñas que no son perceptibles al ojo humano.

Las consecuencias que generan los residuos plásticos van más allá de su disposición final, se centran en el consumidor mismo, quien no se ha formado desde una alfabetización en ciencias, para comprender lo que es la compleja problemática de la contaminación del ambiente,. Este consumidor adolece de educación científica en estos temas, tiene poca concientización; sigue viviendo bajo las antiguas concepciones de que todo lo que se arroja al suelo, al agua o al aire se degrada y vuelve a hacer parte de la tierra, que no hay consecuencia alguna para la dinámica de los ecosistemas y la vida misma.

En el proyecto curricular de Licenciatura en Química de la UPN se desarrollan asignaturas obligatorias en el plan de estudios y algunas líneas de investigación en las que se incorpora la educación ambiental. No obstante, desde diferentes perspectivas como la de González Gaudiano (2001) la forma como se da la educación ambiental en la enseñanza de las ciencias en general y de la química en particular reviste una serie de inconvenientes que se reflejan en activismos que en poco o en nada le aportan a una formación de la ciudadanía de cara a una inminente catástrofe ambiental global. Así mismo, como ninguna aproximación interdisciplinar de las problemáticas socioambientales en las diferentes áreas de la enseñanza de las ciencias.

El conocimiento de los maestros de química frente a cómo contribuir desde su saber científico a mitigar problemáticas socioambientales, es limitado, en cierta medida a la falta de integración de cuestiones ambientales en la enseñanza de la química. De igual manera, la formación del profesorado de química demanda una contigüidad pertinente, seria y adecuada de teorías, modelos y aplicaciones de la 
educación para la sustentabilidad. Desde espacios académicos como; La electiva de Química Verde, es posible el desarrollo de algunas estrategias de orden conceptual y metodológico, en aras de vincular una propuesta centrada en la educación en ciencias para la sustentabilidad. En tal sentido, la biodegradación de un bioplástico por la acción de hongos microscópicos presentes en el suelo, representa una oportunidad para acercarlos, a la construcción de saberes y experiencias tanto científicas como educativas que aporten, a la sustentabilidad del entorno, de la región, país y el planeta, desde la investigación en química, y enseñanza de las ciencias.

\section{Marco teórico}

Una de las mayores problemáticas ambientales es el uso indiscriminado de los polímeros plásticos, parte fundamental del mundo moderno que se utilizan en todo, desde tazas de café hasta automóviles y en la ropa. Así mismo, en medicina su importancia va en aumento para propósitos tan diversos como marcapasos, válvulas cardiacas artificiales y suturas biodegradables (McMurry, 2008). Son estos materiales los que han hecho del mundo moderno el vertedero de basura más grande del planeta, que se acumula día tras día en las calles, en el aire, y en el organismo de muchos seres vivos, llegando al océano y generando un grave problema de contaminación.

Para Tellez (2012), el plástico sintético es un material inorgánico que tiene alta durabilidad; se calcula que puede tardar entre 100 y 1000 años para degradarse dependiendo del tipo. Cada año grandes cantidades de desechos de este material entran al mar desde actividades terrestres y marítimas, tales como la pesca y el turismo, por la pobre gestión de los residuos. La cantidad total en el océano es desconocida porque se han encontrado en todo el mundo, incluso desde las regiones polares hasta el Ecuador, muy lejos de su fuente (United Nations Environment Programme, UNEP, 2014; traducción propia).

Thomson et al. (2005) y Wilber (1987), citado por Tellez (2012), manifiestan que en el centro del océano Pacífico del norte, entre Japón y California, existe una zona denominada "la sopa de plástico", "la isla de la basura" o "el parche de basura del norte", entre otros nombres. Esta zona del océano está cubierta por una gran cantidad de estos desechos. Este fenómeno se da por la existencia de zonas donde el mar está quieto, y por la ausencia de viento y corrientes marinas, haciendo que el agua las arrastre y se acumulen en estas zonas.

Barnes et al. (2009) manifiesta que la presencia de los residuos plásticos representa una amenaza para la biodiversidad marina, debido a que los animales se enredan con estos y pueden resultar heridos, inmóviles o muertos, y estos, con el movimiento de las olas, golpean los corales y los fracturan. Por ende, se terminan fragmentando teniendo consecuencia que pueden ser ingeridos por diferentes especies que los confunden con comida. Esto puede significar la muerte instantánea por ahogamiento o afectar internamente el organismo del animal (Tellez, 2012).

Todos los residuos plásticos, fragmentados pueden absorber "compuestos tóxicos bioacumulativos persistentes" (СTBP) que ya están presentes en el agua y en los sedimentos (Mato et al., 2001; Rios et al., 2007; Macfadyen et al., 2009). Entre los CTBP se incluyen los bifenilos policlorados (PCB), los hidrocarburos poliaromáticos (HPA), el hexaclorociclohexano $(\mathrm{HCH})$ y el insecticida DDT, junto con otros contaminantes orgánicos persistentes (COP) tratados en la Convención de Estocolmo (Stockholm Convention on Persistent Organic Pollutants, 2011). Muchos de estos contaminantes, tienen efectos crónicos para el ser humano como, por ejemplo, trastornos endocrinos que afectan a la reproducción, aumentos en la frecuencia de las mutaciones genéticas (mutagenicidad) y la tendencia a provocar cáncer (carcinogenicidad) (Programa de las Naciones Unidas para el Medio Ambiente, 2011).

Ulloa (2012), citando a Sperling (2006), define que un polímero es una molécula muy grande o macromolécula constituida por la unión repetida de muchas unidades moleculares pequeñas o monómeros, unidas entre sí por enlaces covalentes formadas por reacciones de polimerización. Los polímeros se clasifican según su estructura, composición, origen y tipo de polimerización, sin que estas clasificaciones sean excluyentes entre sí. Existen diferentes términos 
utilizados en la industria de polímeros que corresponden a los diferentes modos de clasificación.

Los biopolímeros o polímeros naturales son aquellos producidos por los seres vivos. Existe una infinidad de polímeros naturales entre los cuales se puede destacar tres grandes grupos: Proteínas, Polisacáridos y Ácidos Nucleícos (Cadena et al., 2000). En efecto, se define un producto plástico como biodegradable si en el plazo máximo de 180 días el $60 \%$ como mínimo de la masa del polímero se descompone en $\mathrm{CO}_{2}$ y $\mathrm{H}_{2} \mathrm{O}$, o bien el $90 \%$ en casi de ser materiales compuestos, sin dejar más del $10 \%$ en peso de residuos después de 12 días de iniciarse el proceso de compostaje (Castells, 2009).

De acuerdo con Dieleman y Juárez (2008), la Unesco establece que la iniciativa de la educación para la sustentabilidad tiene un carácter ambicioso, complejo y reformador, dado el alcance global de la situación social, económica y ambiental que afecta al planeta. Es ambiciosa porque pretende preparar a todas las personas, independientemente de su profesión y condición social, para planificar, enfrentar y resolver las amenazas que pesan sobre la sustentabilidad de nuestro planeta (Unesco, 2005).

La educación para el desarrollo humano sustentable convoca a que las propuestas curriculares se ajusten y se reformen no como algo que se adiciona sino que se debe integrar mediante un proceso de ambientalización curricular o de inclusión de la dimensión ambiental al currículo, de manera innovadora, global y asociada a todos los campos disciplinares de conocimiento, no a uno en concreto, impregnando todas las actividades de las instituciones escolares, para lo cual la formación permanente del profesorado es fundamental (Mora Penagos, 2009).

En consecuencia, esto significa que el profesor debe poseer un perfil amplio que le permita cumplir con las exigencias actuales, reflexionar sobre su propia práctica y ser capaz de asegurar el desarrollo de los valores del medio ambiente y la salud de los escolares. Además, debe garantizar la educación ética y estética, producir transformación científica, actualizar el conocimiento básico general y medio ambiental y evaluar acciones y problemas generales y locales del medio ambiente (Abreu et al., 2009).
La Química Verde es una filosofía de trabajo cuyo objetivo primordial es prevenir la contaminación, no remediarla: reducir al mínimo, o de ser posible, eliminar por completo la contaminación desde su inicio mediante el uso de procesos "limpios", con el fin de evitar al máximo el desperdicio o uso indiscriminado de materias primas no renovables, así como el empleo de materiales peligrosos o contaminantes en la elaboración de productos químicos que no atenten contra la salud o el ambiente (Morales et al. 2011). Del Carmen (2011), citado por Fernández (2013), menciona que el término "trabajos prácticos" se utiliza con frecuencia para referirse a las actividades de enseñanza de las ciencias en las que los alumnos han de utilizar determinados procedimientos para resolverlas. Estos están relacionados con el trabajo de laboratorio o de campo, pero en un sentido más amplio pueden englobar la resolución de problemas científicos o tecnológicos de características diversas. Los trabajos prácticos suponen la articulación de diferentes tipos de actividades, mediante un enfoque integrado, en el que la teoría y la práctica se entrelazan en un tratamiento conjunto.

La finalidad de estos trabajos prácticos, realizados generalmente en el laboratorio, es lograr que el alumno aprenda, en contacto directo con la realidad, la problemática científica inherente a la materia objeto de estudio. Para ello, en el laboratorio, se desarrollarán básicamente tres aspectos: a) una observación detallada del "problema" a estudiar, utilizando el instrumental y las técnicas de cuantificación adecuadas; b) un conocimiento y manejo de instrumentos y técnicas experimentales que permita una valoración adecuado de las mismas, y c) un análisis crítico de los resultados obtenidos. En definitiva, el trabajo de laboratorio debe suponer el ejercicio concreto por parte del alumnado del método de investigación científica (Martínez et al., 2006).

\section{Objetivo general}

- Fomentar principios de educación en ciencias para la sustentabilidad, mediante el diseño, implementación y evaluación de una secuencia didáctica centrada en el desarrollo de trabajos prácticos de laboratorio sobre procesos de biodegradación de bioplásticos, 
a profesores de química en formación inicial del curso electivo de Química Verde de la Universidad Pedagógica Nacional.

\section{Objetivos específicos}

- Indagar por los conocimientos previos de los profesores de química en formación inicial, respecto a la contaminación por plásticos, la contaminación ambiental y la educación en ciencias para la sustentabilidad.

- Diseñar, implementar y evaluar una secuencia didáctica centrada en el desarrollo de trabajos prácticos de laboratorio sobre procesos de biodegradación de bioplásticos.

- Analizar procesos de biodegradación de bioplásticos con profesores de química en formación inicial de la electiva de Química Verde.

- Establecer relaciones entre el abordaje de procesos de biodegradación de bioplásticos, la química verde y la sustentabilidad ambiental.

\section{Metodología}

\section{Población participante}

La población objeto de estudio fueron 21 estudiantes de la Licenciatura en Química (profesores de química en formación inicial, PQFI) y otras licenciaturas, que optaron por cursar el área electiva de Química Verde del Departamento de Química de la Universidad Pedagógica Nacional. Estos se organizaron en cinco grupos de cuatro personas para la implementación y desarrollo de la secuencia didáctica centrada en los TPL (preparación y biodegradación de un bioplástico) y su fomento en la educación en ciencias para la sustentabilidad.

\section{Diseño metodológico.}

Este trabajo se desarrolló en tres etapas en donde se abordaron de manera horizontal tres conceptos (biodegradación, bioplásticos y sustentabilidad en la formación docente) a modo de nodos de análisis.

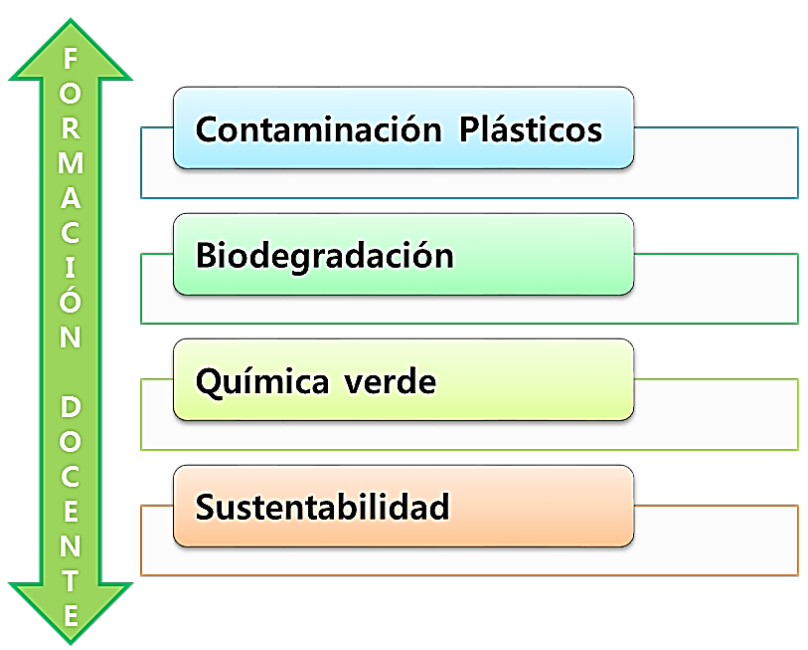

Figura No. 1: Categorías conceptuales y metodológicas para el abordaje de la estrategia didáctica en la formación de profesores. Fuente: elaboración propia.

Cada nodo de aprendizaje esta interrelacionado con las actividades a desarrollar de manera secuencial; por lo tanto, los recursos de indagación, los conversatorios, talleres y trabajos prácticos de laboratorio están sujetos al desarrollo de la educación en ciencias para la sustentabilidad en la química verde.

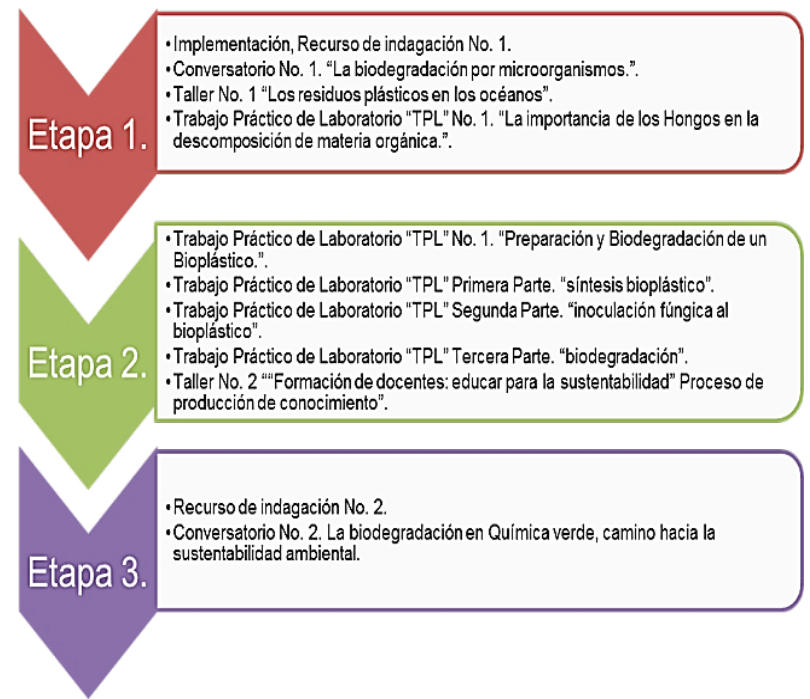

Figura No. 2: Etapas para el abordaje de la estrategia didáctica en la formación de profesores de química. Fuente: elaboración propia.

\section{Resultados}

Para el análisis y la correspondiente discusión de los resultados se optó por la estructuración de nodos 
de análisis, pues estos, al interconectarse a manera de red (Franco dos Santos y Franco Moreno, 2014), propician elementos claros y de rigurosidad en el tratamiento de la información recopilada y su interrelación de conformidad con el problemática identificada (contaminación por plásticos) y la metodología utilizada. Dichos nodos se presentan a continuación (ver tabla 5):

Tabla 5. Nodos y criterios de análisis de la información

\begin{tabular}{|c|c|c|}
\hline Nodos de análisis & Criterios a considerar & $\begin{array}{c}\text { Insumos de } \\
\text { información }\end{array}$ \\
\hline $\begin{array}{c}\text { 1. El estudio de los procesos de } \\
\text { biodegradación de plásticos y su relación } \\
\text { con la sustentabilidad ambiental. }\end{array}$ & $\begin{array}{c}\text { Abordaje teórico y práctico de conceptos asociados a } \\
\text { los plásticos y a los procesos de biodegradación. }\end{array}$ & $\begin{array}{c}\text { T.P.L 1, } 2 \text { y 3. } \\
\text { Talleres } 1 \text { y } 2 .\end{array}$ \\
\hline $\begin{array}{c}\text { 2. Incidencia de la secuencia didáctica. } \\
\text { Desempeño y posicionamiento de los P.Q.F.l. frente } \\
\text { a los problemas socioambientales en relación con los } \\
\text { plásticos y su relación con la Química verde. }\end{array}$ & $\begin{array}{c}\text { Pruebas tipo Likert } \\
1 \text { y } 2 .\end{array}$ \\
$\begin{array}{c}\text { Conversatorio } 1 \text { y } 2 . \\
\text { verde para la sustentabilidad ambiental } \\
\text { en la formación de profesores de ciencias. }\end{array}$ & $\begin{array}{c}\text { Perspectiva crítica y reflexiva del profesor } \\
\text { investigador en relación aspectos centrales de los } \\
\text { nodos 1 y } 2 \text { (interconexión). }\end{array}$ & $\begin{array}{c}\text { Secuencia didáctica } \\
\text { implementada. }\end{array}$ \\
\hline
\end{tabular}

Fuente: elaboración propia

\section{Conclusiones}

A partir del proceso de biodegradación de bioplásticos centrado en el desarrollo de trabajos prácticos de laboratorio, es posible fomentar una educación en ciencias para la sustentabilidad, ya que a través de la actividad experimental el pqfi aborda los problemas ambientales de manera inter y transdisciplinar, donde el desarrollo de estos favorecerá el aprendizaje de conceptos y procedimientos.

El desarrollo de secuencias didácticas enfocadas en la educación sustentable permiten que el PQFI adquiera destrezas frente a como contribuir desde su profesión docente a mitigar las problemáticas ambientales, a través del desarrollo de micro-proyectos pro-ambientales.

La integración de la sustentabilidad ambiental al programa de Licenciatura en Química en general, la formación de profesores de ciencias en particular, y su relación con la química verde está encaminada al desarrollo de competencias genéricas y profesionales del futuro docente para que sea capaz de identificar e intervenir, desde la perspectiva educativa, en la problemática ambiental.

\section{Referencias}

Abreu Santos y Villalón Legrá. (2009). La educación ambiental para el desarrollo sostenible desde la gestión, investigación e innovación educativa en la formación y desempeño del profesional de la educación.Cub@: Medio ambiente y DesarroIlo. Revista electrónica de la Agencia de Medio Ambiente, (17). Recuperado de http://ama.redciencia.cu/articulos/17.06.pdf
Barnes, D. A., Galgani, F., Thomson, R. C., y Barlaz, M. (2009). Accumulation and Fragmentation of Plastic Debris in Global Environments. Philosophical Transactions of the Royal Society Biological Sciences, (364), 1985-1998.

Cadena F., y Quiroz F. (2000). Manual de reciclaje de plásticos. Quito: Corporación Oikos. 
Castells, X. (2009). Reciclaje de residuos industriales; residuos sólidos urbanos y fangos de depura. Madrid: Ediciones Díaz de Santos.

Dieleman, H., y Najera, J. (2008). ¿Cómo se puede diseñar educación para la sustentabilidad? Revista Internacional de Contaminacion Ambiental, 24(3), 131-147.

Fernández, N. (2013). Los Trabajos Prácticos de Laboratorio por investigación en la enseñanza de la Biología. Revista de Educación en Biología, 16(2), 15-30.

Franco dos Santos, P., y Franco, R. (2014). Rol de los participantes del programa colombo-brasileño de formación de profesores en la interfaz universidad-escuela: discusión sobre aspectos teóricos asociados. En L. Martínez y D. Parga (Coords.), Formación permanente de profesores en la interfaz universidad-escuela: currículo, fundamentos y roles. una experiencia en construcción. Bogotá: Universidad Pedagógica Nacional.

González, E. (2001). Otra lectura a la historia de la educación ambiental en América Latina. Desenvolvimento e Meio Ambiente, (3), 141-158.

González-Viñas, W., y Mancini, H. (2003). Ciencia de los materiales. Barcelona: Ariel Ciencia y Tecnología.

Gunther, M. (2006, 2 de noviembre) Why it's not easy being green. Recyclers go after 'natural' firm, NatureWorks. Fortune's Marc Gunther reports. Fortune. Recuperado de http://money.cnn.com/2006/11/01/ news/companies/pluggedin_gunther_natureworks. fortune/index.htm?postversion=2006110207

Macfadyen, G., Huntington, T., y Cappell, R. (2009). Abandoned, lost or otherwise discarded fishing gear (FAO Fisheries and Aquaculture Technical Papers). Roma: United Nations Environment Programme, Food and Agriculture Organization of the United Nations.

Martínez, E., y Agustí, L., (2006). Prácticas de crecimiento y desarrollo de los vegetales. Barcelona: Universitat de Barcelona.

Mato, Y., Isobe, T., Takada, H., Kanehiro, H., Ohtake, C., y Kaminuma, T. (2001). Plastic Resin Pellets as a Transport Medium for Toxic Chemicals in the Marine Environment. Environmental Science and Technology, 35(3), 318-324.
McMurry, J. (2008). Química orgánica. México, D.F.: Cengage Learning.

Mora, W. M. (2009). Educación ambiental y educación para el desarrollo sostenible ante la crisis planetaria: demandas a los procesos formativos del profesorado. Tecné, Episteme y Didaxis, (26), 7-35.

Morales G. M., Martínez J. O., Reyes S. L., Hernández, O. M., Arroyo R. G., Valdivia, A. O., Miranda R. R. (2011). ¿Qué tan verde es un experimento? Educación Química, 22(3), 240-248.

Programa de las Naciones Unidas para el Medio Ambiente. (2011). Anuario PNUMA: temas emergentes en nuestro medio ambiente global: Residuos plásticos en los océanos. Nairobi, Kenya: Programa de las Naciones Unidas para el Medio Ambiente, UNEP.

Rios, L. M., Moore, C., y Jones, P. R. (2007). Persistent Organic Pollutants Carried by Synthetic Polymers in the Ocean Environment. Marine Pollution Bulletin, $54,1230-1237$.

Sperling, L. H. (2006). Introduction to Physical Polymer Science. Nueva Jersey: Wiley-Interscience.

Stockholm Convention on Organic Pollutants. (2011). What are POPs? Recuperado de http://www.pops.int

Tellez, M. A. (2012). La complejidad de la problemática ambiental de los residuos plásticos: una aproximación al análisis narrativo de política pública en Bogotá (Tesis inédita de magister). Universidad Nacional de Colombia, Bogotá.

Ulloa, M. J. (2012). Desarrollo de biopolímeros a partir de almidón de corteza de yuca. (Tesis inédita de pregrado). Universidad Técnica Particular de Loja, Ecuador.

Unesco. (2005). Report by the Director-General on the United Nations of Education for Sustainable Development: Draft International Implementation Scheme and Unesco's contribution to the implementation of the Decade (2005-2014). Hundred and seventy-second session. Paris, August 2005. Recuperado de http://www.unesco.org/ education/desd

Wilber, R. J. (1987). Plastics in the North Atlantic. Oceanus, 30(3), 61-68. 